\title{
THE FUKUMOTO-FURUTA AND THE OZSVÁTH-SZABÓ INVARIANTS FOR SPHERICAL 3-MANIFOLDS
}

\author{
MASAAKI UE \\ Department of Mathematics, Kyoto University \\ Kyoto, 606-8502, Japan \\ E-mail: ue@math.kyoto-u.ac.jp \\ Dedicated to Professor Takao Matumoto for his 60th birthday
}

\begin{abstract}
We show that the Fukumoto-Furuta invariant for a rational homology 3-sphere $M$, which coincides with the Neumann-Siebenmann invariant for a Seifert rational homology 3-sphere, is the same as the Ozsváth-Szabó's correction term derived from the Heegaard Floer homology theory if $M$ is a spherical 3-manifold.
\end{abstract}

1. Introduction. Let $X$ be an oriented compact smooth 4-manifold and $I_{X}$ be its intersection form. In the case where $X$ is closed, various deep results about the constraints on $I_{X}$ have been obtained by gauge theory and Seiberg-Witten theory (see [1], [3]). To obtain similar results in the case where $X$ has a boundary, we need extra invariants of a 3 manifold that bounds $X$. As one of such invariants we have studied the Fukumoto-Furuta invariant $w\left(M, X, c_{X}\right)$ for a triple $\left(M, X, c_{X}\right)([5],[18],[19])$, where $M$ is a closed oriented 3-manifold, $X$ is a compact spin 4-orbifold with $\partial X=M$, and $c_{X}$ is a spin structure on $X$. In [19] we have shown that if $M$ is a Seifert rational homology 3-sphere, there exists a canonical choice of $X$ so that $w\left(M, X, c_{X}\right)$ is the same as the Neumann-Siebenmann invariant $\bar{\mu}\left(M, c_{M}\right)$, determined only by $M$ and a spin structure $c_{M}$ on $M$, which is induced by $c_{X}$.

Another powerful invariant giving several constraints on the intersection forms of 4manifolds with boundary is given by Ozsváth-Szabó's correction term $d(M, t)$ defined for a rational homology 3 -sphere $M$ with $\operatorname{spin}^{c}$ structure $t$. These two invariants are derived from different theories (the Seiberg-Witten theory and the Heegaard Floer homology theory) and they are not the same even if $M$ is a Seifert rational homology 3-sphere and

2000 Mathematics Subject Classification: Primary 57M27; Secondary 57N13, 57N10.

Key words and phrases: spherical 3-manifolds, Ozsváth-Szabó invariant, Fukumoto-Furuta invariant.

The paper is in final form and no version of it will be published elsewhere. 
$t$ comes from a spin structure. However we can show that they are the same if $M$ is a spherical 3-manifold. In fact in $\S 3.1$ we extend the definition of the Fukumoto-Furuta invariant $w\left(M, X, t_{X}\right)$ for a triple $\left(M, X, t_{X}\right)$, where $M$ and $X$ are as before and $t_{X}$ is a $\operatorname{spin}^{c}$ structure on $X$. If $M$ is spherical, we can also define this invariant so that it depends only on $M$ and the $\operatorname{spin}^{c}$ structure on $M$ by choosing the cone over $M$ as $X$. The main result of this paper is the following theorem, whose proof will be given in $\S 3$.

THEOREM 1.1. Let $(S, t)$ be a pair of a spherical 3-manifold and a $\operatorname{spin}^{c}$ structure on it. Then $4 d(S, t)=-\bar{\mu}(S, t)$. Here $\bar{\mu}(S, t)$ is the Fukumoto-Furuta invariant $w\left(S, c S, t_{c S}\right)$, where $c S$ is the cone over $S$ and $t_{c S}$ is the unique $\operatorname{spin}^{c}$ structure that is an extension of $t$. In particular if $t$ is a spin structure, then $-4 d(S, t)$ coincides with the NeumannSiebenmann invariant, which is a spin rational homology cobordism invariant whose value modulo 16 is the Rokhlin invariant of $(S, t)$.

We note that the above claim does not hold for a non-spherical Seifert rational homology 3-sphere with spin structure. In fact $d(M, c)=0$ but $\bar{\mu}(M, c)=8$ if $M$ is the Brieskorn homology 3 -sphere $\Sigma(2,3,7)$ and $c$ is the unique spin structure on it. This implies that $-4 d(M, c)(\bmod 16)$ is not the Rokhlin invariant in general, in spite of the rational homology cobordism invariance of $d(M, c)([11])$.

In this paper we only consider the Seifert 3-manifolds over orientable base 2-orbifolds. In $\S 1$ and $\S 2$ we recall the definition and several properties of $w\left(M, X, c_{X}\right)$ and $d(M, t)$ respectively. In $\S 1$ we also consider a Seifert rational homology 3 -sphere $M$ with spin structure $c$ bounding a negative definite spin 4-manifold $W$ and give a constraint on $b_{2}(W)$ in terms of $\bar{\mu}(M, c)$, which is deduced from the orbifold 10/8-theorem and its refinement. In $\S 3.4$ we compare this result with the constraint given by $d(M, c)$ in [11]. In $\S 3.4$ we give the explicit formula for the above invariants for $L(p, q)$ and the correspondence between the description of the $\operatorname{spin}^{c}$ structures on $L(p, q)$ given in [11] and ours given in $\S 3$. In $\S 4$ we give some remarks on the case of plumbed 3-manifolds.

2. The Fukumoto-Furuta invariants. We start with a rational homology 3 -sphere $M$ with spin structure $c$. For such a pair $(M, c)$, there always exists a compact spin 4-orbifold $X$ with spin structure $c_{X}$, such that $\partial X=M$ and $c_{X}$ restricted on $M$ induces $c$. Let us recall the definition of the Fukumoto-Furuta invariant for a triple $\left(M, X, c_{X}\right)$, which is slightly different from the original one for an integral homology 3-sphere in [5].

Definition 2.1. For $\left(M, X, c_{X}\right)$ given above, there exists a spin 4-manifold $Y$ with spin structure $c_{Y}$ such that $\partial\left(Y, c_{Y}\right)=(M, c)$, where $c$ is the restriction of $c_{X}$ on $M$. Then for any choice of such $\left(Y, c_{Y}\right)$, we define the Fukumoto-Furuta invariant by

$$
w\left(M, X, c_{X}\right)=-8 \operatorname{ind} \mathcal{D}(X \cup(-Y))+\sigma(Y) .
$$

Here $\sigma(Y)$ is the signature of $Y, \mathcal{D}(X \cup(-Y))$ is the spin Dirac operator defined over a closed spin 4-orbifold $X \cup(-Y)$ whose spin structure is induced by $c_{X}$ and $c_{Y}$, and ind denotes the index over $\mathbf{C}$.

In this paper we always assume for simplicity that

- $b_{1}(X)=0$, and

- the set $\Sigma X$ of singularities of $X$ consists only of isolated points. 
Then $w\left(M, X, c_{X}\right)$ does not depend on the choice of $\left(Y, c_{Y}\right)$ by the excision principle or explicit computation of the index below. Moreover $w\left(M, X . c_{X}\right)(\bmod 16)$ is equal to the Rokhlin invariant of $(M, c)$ since ind $\mathcal{D}$ is even for a spin 4-orbifold.

Several important properties of the Fukumoto-Furuta invariant are derived from the following theorem.

THEOREM 2.2 (The orbifold 10/8-theorem [4]). Let $Z$ be a closed spin 4-orbifold with $b_{1}(Z)=0$. Then

1. Either $\operatorname{ind} \mathcal{D}(Z)=0$ or

$$
1-b_{2}^{-}(Z) \leq \operatorname{ind} \mathcal{D}(Z) \leq b_{2}^{+}(Z)-1 .
$$

2. In particular if $b_{2}^{ \pm}(Z) \leq 2$, then ind $\mathcal{D}(Z)=0$ since ind $\mathcal{D}(Z)$ is even.

We can compute $\mathcal{D}(Z)$ (and $w\left(M, X, c_{X}\right)$ ) by using the Kawasaki V-index theorem [7], which gives the following results in our case. Here we also assume that the set of singularities $\Sigma Z$ of $Z$ consists only of isolated ones.

$$
\begin{aligned}
& \operatorname{ind} \mathcal{D}(Z)=-\frac{1}{24} \int_{Z} p_{1}+\delta^{\text {Dirac }}(Z), \\
& \sigma(Z)=\frac{1}{3} \int_{Z} p_{1}+\delta^{\operatorname{sign}}(Z) .
\end{aligned}
$$

Here $\delta^{\text {Dirac }}(Z)$ (resp. $\delta^{\text {sign }}(Z)$ ) denotes the sum of the contribution $\delta^{\text {Dirac }}(x)$ (resp. $\left.\delta^{\operatorname{sign}}(x)\right)$ from the isolated singularities $x \in \Sigma Z$ to the index of the Dirac (resp. the signature) operator over $Z$. Thus

$$
\operatorname{ind} \mathcal{D}(Z)=-\frac{1}{8}\left(\sigma(Z)+\sum_{x \in \Sigma Z} \delta(x)\right),
$$

where

$$
\delta(x)=-8 \delta^{\operatorname{Dirac}}(x)-\delta^{\operatorname{sign}}(x) .
$$

Here a neighborhood of $x$ is a cone $c S$ over a spherical 3-manifold $S$. Then there is a one-to-one correspondence between the set of spin structures on $S$ and the set of spin structures on $c S$ [18]. Moreover $\delta(x)$ is determined uniquely by $\left(S, c_{S}\right)$ and hence is denoted by $\delta\left(S, c_{S}\right)$, where $c_{S}$ is a spin structure on $S$ induced by $c_{Z}$. Thus $w(M, X, c)$ is represented as follows.

Proposition 2.3 ([19]).

$$
w(M, X, c)=\sigma(X)+\delta(X, c)
$$

with

$$
\delta(X, c)=\sum_{S} \delta\left(S, c_{S}\right)
$$

where in the sum on the right hand side $S$ runs over all the links of the isolated singularities of $X$ and $c_{S}$ denotes the restriction of $c$ to $S$.

Remark 2.4. The above $\delta(S, c)$ is equal to the Fukumoto-Furuta invariant $w\left(S, c S, c_{c S}\right)$, where $c_{c S}$ is the unique spin structure on $c S$ whose restriction to $S$ is $c$.

The list of the values of $\delta(S, c)$ for $(S, c)$ is given explicitly in [18]. 
(1) The case when $S=L(p, q)$.

In the case when $S$ is a lens space $L(p, q)$, which is the $-p / q$-surgery on the unknot in $S^{3}$, we describe a spin structure $c$ on $L(p, q)$ by assigning a $\mathbf{Z}_{2}$ value $c(\mu)$ to the meridian $\mu$ of the unknot satisfying

$$
p c(\mu)+p q \equiv 0 \quad(\bmod 2) .
$$

This equation shows that $c(\mu) \equiv q(\bmod 2)$ if $p$ is odd, but $c(\mu)$ may be arbitrary if $p$ is even. The value $c(\mu)$ determines a spin structure on the complement of the unknot $\left(c(\mu)=0\right.$ if and only if $c$ extends to the spin structure on $\left.S^{3}\right)$, and the above equation is equivalent to the condition for this spin structure to be extendable to that on the solid torus attached by the $-p / q$-surgery. Then $\delta(L(p, q), c)$ is described by a $\sigma$-function as follows. (We note that either $p$ or $q$ may be negative according to the above convention.) Proposition $2.5([5])$.

$$
\delta(L(p, q), c)=\sigma\left(q, p,(-1)^{c(\mu)-1}\right),
$$

where $\sigma(q, p, \epsilon)(\operatorname{gcd}(p, q)=1, \epsilon= \pm 1)$ is uniquely determined by the following formula.

1. $\sigma(q+k p, p, \epsilon)=\sigma\left(q, p,(-1)^{k} \epsilon\right)$,

2. $\sigma(-q, p, \epsilon)=\sigma(q,-p, \epsilon)=-\sigma(q, p, \epsilon)$,

3. $\sigma(q, 1, \epsilon)=0$,

4. $\sigma(p, q,-1)+\sigma(q, p,-1)=-\operatorname{sgn} p q$ if $p+q \equiv 1(\bmod 2)$.

According to the $\mathrm{V}$-index theorem $\sigma$ is described as ([5])

$$
\sigma(q, p, \epsilon)=\frac{1}{p} \sum_{k=1}^{|p|-1}\left(\cot \frac{\pi k}{p} \cot \frac{\pi k q}{p}+2 \epsilon^{k} \csc \frac{\pi k}{p} \csc \frac{\pi k q}{p}\right) .
$$

We also note that if $p$ is odd, we only consider $\sigma(q, p, \epsilon)$ for the case when $\epsilon=(-1)^{q-1}$.

(2) The case when $S \neq L(p, q)$.

In this case we give a complete list of $\delta(S, c)$ in [18]. In the case when $S$ is a Seifert fibration over the 2-orbifold of type $S^{2}(2,2, n)$, then $\delta(S, c)$ is described in terms of the $\sigma$-function above, and $\delta(S, c)$ is given more explicitly otherwise.

If $(M, c)$ is a Seifert rational homology 3-sphere with spin structure, we show in [19] that $\left(X, c_{X}\right)$ can be chosen so that $w\left(M, X, c_{X}\right)$ is equal to the Neumann-Siebenmann invariant $\bar{\mu}(M, c)$ (and hence it depends only on $(M, c)$ ), which is defined as follows.

Definition 2.6. Let $P(\Gamma)$ be a plumbing associated with an integrally weighted tree $\Gamma$. Each vertex $v$ of $\Gamma$ with weight $e$ corresponds to the $D^{2}$-bundle over $S^{2}$ with Euler class $e$ contained in $P(\Gamma)$ whose zero section generates $H_{2}(P(\Gamma), \mathbf{Z})$, which we denote by $[v]$. Let $M=\partial P(\Gamma)$ and $c$ be a spin structure on $M$. Then there exists a unique element of the form

such that

$$
w(P(\Gamma), c)=\sum_{v} \epsilon_{v}[v] \in H_{2}(P(\Gamma), \mathbf{Z})
$$

1. each $\epsilon_{v}$ is either 0 or 1 , and

2. the Poincaré dual of $w(P(\Gamma), c) \bmod 2$ is the obstruction to extending $c$ to the spin structure on $P(\Gamma)$. 
Then the Neumann-Siebenmann invariant of $(M, c)$ is defined to be

$$
\bar{\mu}(M, c)=\sigma(P(\Gamma))-w(P(\Gamma), c) \cdot w(P(\Gamma), c) .
$$

The value of $\bar{\mu}(M, c)$ depends only on $(M, c)$ (does not depend on the choice of $P(\Gamma)$ with $M=\partial P(\Gamma))$.

In case of a Seifert rational homology 3-sphere $M$ with spin structure $c$, we always find a star-shaped weighted graph $\Gamma$ with $M=\partial P(\Gamma)$, and the Fukumoto-Furuta invariant and $\bar{\mu}$ are related as follows.

Lemma 2.7 ([19]). Let $(M, c)$ be a pair of a Seifert rational homology 3-sphere and a spin structure on it. Then there exist two compact spin 4-orbifolds with only isolated singularities $X_{ \pm}$with spin structures $c_{ \pm}$such that

1. $\partial\left(X_{ \pm}, c_{ \pm}\right)=(M, c)$,

2. $w\left(M, X_{+}, c_{+}\right)=w\left(M, X_{-}, c_{-}\right)=\bar{\mu}(M, c)$,

3. $b_{1}\left(X_{+}\right)=b_{1}\left(X_{-}\right)=0, b_{2}^{+}\left(X_{+}\right) \leq 1$, and $b_{2}^{-}\left(X_{-}\right) \leq 1$.

Here we note that $w\left(M, X_{ \pm}, c_{ \pm}\right)=\sigma\left(X_{ \pm}\right)+\delta\left(X_{ \pm}, c_{ \pm}\right)$.

Combining this lemma with the orbifold 10/8-theorem we proved the following theorem in [19], which extends the theorem by Saveliev for Seifert integral homology 3-spheres $[15]$.

THEOREM 2.8 ([19]). Let $\left(M_{i}, c_{i}\right)(i=1,2)$ be Seifert rational homology 3-spheres with spin structure. If there exists a spin cobordism $(W, c)$ from $\left(M_{1}, c_{1}\right)$ to $\left(M_{2}, c_{2}\right)$ with $b_{2}(W)=0$, then $\bar{\mu}\left(M_{1}, c_{1}\right)=\bar{\mu}\left(M_{2}, c_{2}\right)$.

We also obtain the following constraint on the intersection form of a spin 4-manifold bounded by a Seifert rational homology 3 -sphere.

THEOREM 2.9. Suppose that a Seifert rational homology 3-sphere $M$ with spin structure $c$ bounds a negative definite spin 4-manifold $Y$ with spin structure $c_{Y}$. Then

$$
\begin{aligned}
& b_{2}(Y) \equiv-\bar{\mu}(M, c) \quad(\bmod 16), \\
& -\frac{\bar{\mu}(M, c)}{9} \leq b_{2}(Y) \leq-\bar{\mu}(M, c) .
\end{aligned}
$$

Proof. We choose two spin 4-orbifolds $\left(X_{ \pm}, c_{ \pm}\right)$bounded by $(M, c)$ as in Lemma 2.7 so that

$$
\bar{\mu}(M, c)=w\left(M, X_{ \pm}, c_{ \pm}\right) .
$$

Furthermore by applying the formula stated above to a closed spin 4-orbifold $\left(Z_{ \pm}, c_{Z_{ \pm}}\right)=$ $\left(X_{ \pm}, c_{X_{ \pm}}\right) \cup\left(-Y, c_{Y}\right)$ we have

$$
w\left(M, X_{ \pm}, c_{ \pm}\right)=-8 \operatorname{ind} \mathcal{D}\left(Z_{ \pm}\right)+\sigma(Y)=-8 \operatorname{ind} \mathcal{D}\left(Z_{ \pm}\right)-b_{2}(Y) .
$$

Thus since

$$
\text { ind } \mathcal{D}\left(Z_{ \pm}\right)=-\frac{1}{8}\left(\bar{\mu}(M, c)+b_{2}(Y)\right)
$$

is even, we have the first formula above. Next applying the orbifold $10 / 8$-theorem to $Z_{ \pm}$ we have either

$$
\operatorname{ind} \mathcal{D}\left(Z_{ \pm}\right)=0
$$


in which case $b_{2}(Y)=-\bar{\mu}(M, c)$, or

$$
1-b_{2}^{-}\left(Z_{ \pm}\right) \leq \operatorname{ind}\left(Z_{ \pm}\right) \leq b_{2}^{+}\left(Z_{ \pm}\right)-1 .
$$

Here we note that

$$
b_{2}^{+}\left(Z_{+}\right)=b_{2}^{+}\left(X_{+}\right)+b_{2}^{-}(Y) \leq 1+b_{2}(Y) \text {, }
$$

and

$$
b_{2}^{-}\left(Z_{-}\right)=b_{2}^{-}\left(X_{-}\right)+b_{2}^{+}(Y) \leq 1 .
$$

It follows that

$$
0 \leq-\bar{\mu}(M, c)-b_{2}(Y) \leq 8 b_{2}(Y),
$$

which gives the second formula

We can slightly strengthen the above result by applying the following refined inequality for the index of the Dirac operator, which is the orbifold version of the theorem in $[6]$.

THEOREM $2.10([6])$. Let $Z$ be a closed spin 4-orbifold with $b_{1}(Z)=0$. Then either ind $\mathcal{D}(Z)=0$ or

$$
\epsilon\left(-\frac{1}{2} \operatorname{ind} \mathcal{D}(Z), b_{2}^{-}(Z)\right)-b_{2}^{-}(Z) \leq \operatorname{ind} \mathcal{D}(Z) \leq b_{2}^{+}(Z)-\epsilon\left(\frac{1}{2} \operatorname{ind} \mathcal{D}(Z), b_{2}^{+}(Z)\right)
$$

where $\epsilon(d, \ell)$ is determined by the following rule.

$$
\epsilon(d, \ell)=\left\{\begin{array}{lll}
3 & (d \equiv 0 & \bmod 4, \ell \geq 4), \\
1 & (d \equiv 0 & \bmod 4, \ell<4), \\
1 & (d \equiv 1 & \bmod 4) \\
2 & (d \equiv 2 & \bmod 4) \\
3 & (d \equiv 3 & \bmod 4)
\end{array}\right.
$$

The following result is obtained by modifying the proof of Theorem 2.9 , so we omit the proof.

TheOREm 2.11. Suppose that a Seifert rational homology 3-sphere $M$ with spin structure $c$ bounds a negative definite spin 4-manifold $Y$. We put $d=-\left(\bar{\mu}(M, c)+b_{2}(Y)\right) / 16$, which is an integer by the first claim of Theorem 2.9. Then we have either $b_{2}(Y)=-\bar{\mu}(M, c)$ or

$$
\frac{1}{9}(-\bar{\mu}(M, c)+8(\epsilon-1)) \leq b_{2}(Y) \leq-\bar{\mu}(M, c)-8\left(\epsilon^{\prime}-1\right),
$$

where

$$
\left(\epsilon, \epsilon^{\prime}\right)=\left\{\begin{array}{lll}
(1,1) & \text { if } d \equiv 0 \quad \bmod 4 \\
(1,3) & \text { if } d \equiv 1 \quad \bmod 4 \\
(2,2) & \text { if } d \equiv 2 \bmod 4 \\
(3,1) & \text { if } d \equiv 3 \bmod 4 .
\end{array}\right.
$$

If $M$ is a spherical 3-manifold we can replace the above inequality by

$$
\frac{1}{9}(-\bar{\mu}(M, c)+8 \epsilon) \leq b_{2}(Y) \leq-\bar{\mu}(M, c)-8 \epsilon^{\prime}
$$

for the same $\left(\epsilon, \epsilon^{\prime}\right)$. 
3. The Ozsváth-Szabó invariant. In this section we recall the definition and some properties of the Ozsváth-Szabó's correction term. Let $M$ be a rational homology 3-sphere with $\operatorname{spin}^{c}$ structure $t$. In [10] Ozsváth and Szabó defined the Heegaard Floer homologies $H F^{+}, H F^{\infty}$, and $H F_{\text {red }}$ for $(M, t)$. Here $H F^{+}(M, t)$ has an absolute grading with value in $\mathbf{Q}$, and $H F_{\text {red }}(M, t)$, which is the cokernel of the natural map from $H F^{\infty}(M, t)$ to $H F^{+}(M, t)$, is a finitely generated abelian group with an absolute $\mathbf{Z}_{2}$ grading and hence its Euler chracteristic $\chi\left(H F_{\text {red }}(M, t)\right)$ is well-defined.

Definition 3.1 ([11]). The correction term $d(M, t)$ is defined to be the minimum of the absolute grading of the elements in $\operatorname{Im}: H F^{\infty}(M, t) \rightarrow H F^{+}(M, t)$.

In [11] Ozsváth and Szabó proved several properties of $d(M, t)$. For example, $d$ changes the sign if the orientation of $M$ is reversed, $d(M, t)=d(M, \bar{t})$ where $\bar{t}$ is the conjugation of $t$, and $d$ is additive under a connected sum operation. Furthermore they proved the following results.

THEOREM $3.2([11])$.

1. Let $\left(M_{i}, t_{i}\right)(i=1,2)$ be a rational homology 3-sphere with $\operatorname{spin}^{c}$ structure. Suppose that there exists a $\operatorname{spin}^{c}$ rational homology cobordism $\left(W, t_{W}\right)$ from $\left(M_{1}, t_{1}\right)$ to $\left(M_{2}, t_{2}\right)$. Then $d\left(M_{1}, t_{1}\right)=d\left(M_{2}, t_{2}\right)$.

2. If $(M, t)$ bounds a 4-manifold $\left(W, t_{W}\right)$ with $\operatorname{spin}^{c}$ structure $t_{W}$, then

$$
d(M, t) \equiv \frac{c_{1}\left(t_{W}\right)^{2}-\sigma(W)}{4} \quad(\bmod 2 \mathbf{Z}) .
$$

3. If $(M, t)$ bounds a negative definite 4-manifold $\left(W, t_{W}\right)$ with $\operatorname{spin}^{c}$ structure $t_{W}$, then

$$
c_{1}\left(t_{W}\right)^{2}+b_{2}(W) \leq 4 d(M, t)
$$

Rustamov [14] (together with the result by Nicolaescu [13]) gives the relations between the correction term $d(M, t)$ and the modified Seiberg-Witten invariant for $(M, t)$ via the modified Reidemeister torsion as follows. To describe his statement we introduce several notations.

Definition 3.3. For a rational homology 3 -sphere with $\operatorname{spin}^{c} \operatorname{structure}(M, t)$, we put

$$
\xi^{0}(M, t)=\frac{1}{2} d(M, t)-\chi\left(H F_{\text {red }}(M, t)\right) .
$$

The sign convention is opposite to that in [14]. In addition we choose a Riemann metric $g$ of $M$ and let $s w(M, t)$ be the 3-dimensional Seiberg-Witten invariant for $(M, t)$ with respect to $g$ (the number of the irreducible solutions to the Seiberg-Witten equation counted with sign). We also denote by $\eta^{\text {Dirac }}(M, t)$ (resp. $\left.\eta^{\text {sign }}(M)\right)$ the eta invariant of the Dirac (resp. the signature) operator with respect to $g$. Then we define the modified Seiberg-Witten invariant to be

$$
s w^{0}(M, t)=s w(M, t)+\frac{1}{8}\left(4 \eta^{\operatorname{Dirac}}(M, t)+\eta^{\text {sign }}(M)\right) .
$$

The value of $s w^{0}(M, t)$ does not depend on the choice of $g$. 
TheOREm $3.4([14]) \cdot \xi^{0}(M, t)=s w^{0}(M, t)$. In fact the both invariants are equal to the modified Reidemeister torsion

$$
\tau^{0}(M, t)=\tau(M, t)+\frac{1}{2}\left|H_{1}(M, \mathbf{Z})\right| \lambda(M)
$$

where $\tau(M, t)$ is the Reidemeister-Turaev torsion of $(M, t)$ and $\lambda(M)$ is the CassonWalker invariant of $M$. (The equality $\tau^{0}=s w^{0}$ is proved in [13].)

4. Proof of Theorem 1.1. In this section we focus on the Fukumoto-Furuta invariant for a spherical 3-manifold.

4.1. The Fukumoto-Furuta invariant for $\operatorname{spin}^{c}$ structures. We discuss the FukumotoFuruta invariant for a triple $\left(M, X, t_{X}\right)$, where $M, X$ are as before and $t_{X}$ is a spin ${ }^{c}$ structure on $X$. We can define $w\left(M, X, t_{X}\right)$ for such a triple as in Definition 2.1 by choosing a 4-manifold $Y$ with $\operatorname{spin}^{c}$ structure $t_{Y}$ such that $\partial\left(Y, t_{Y}\right)=\left(M,\left.t_{X}\right|_{M}\right)$. We note that $M$ always bounds a 1-connected spin 4-manifold $Y$, in which case the inclusion $M \rightarrow Y$ induces the surjection $H^{2}(Y, \mathbf{Z}) \rightarrow H^{2}(M, \mathbf{Z})$. It follows that any given $\operatorname{spin}^{c}$ structure on $M$ extends to that on $Y$. However we can also define $w\left(M, X, t_{X}\right)$ without any auxiliary manifold as follows. First we start with a spherical 3-manifold $S$ with spin ${ }^{c}$ structure $t$. We write $S=S^{3} / G$, where $G$ is a finite subgroup of $S O(4)$ acting freely on $S^{3}$. Up to conjugacy we can assume that $G$ is a subgroup of $U(2)=S^{3} \times_{\mathbf{z}_{2}} S^{1}$. Then $c S$ is represented as $D^{4} / G$. Next we fix a spin structure $c_{S}$ on $S$, which extends uniquely to the spin structure $c_{c S}$ on $c S$ [19]. We note that $c_{c S}$ is determined by the choice of the double covering $\sqrt{K}$ of the canonical bundle $K$ over $c S$. We denote by $P_{\operatorname{spin}(4)}$ the corresponding orbifold principal spin(4)-bundle over $c S$ that covers the frame bundle over $c S$. The difference between $t$ and $c_{S}$ is represented by a complex line bundle $L=S^{3} \times{ }_{\rho} \mathbf{C}$ over $S$, which is a flat bundle associated with some representation $\rho: G \rightarrow U(1)$. Note that $\rho$ corresponds to $c_{1}(L) \in H^{2}(S, \mathbf{Z})$ via the isomorphism $H^{2}(S, \mathbf{Z}) \cong \operatorname{Ext}\left(H_{1}(S, \mathbf{Z}), \mathbf{Z}\right) \cong$ $\operatorname{Hom}(G, U(1))$. Then $L$ extends to the orbifold line bundle $\widetilde{L}=D^{4} \times{ }_{\rho} \mathbf{C}$ over $c S$, and using $P_{\text {spin(4) }}$ and the orbifold principal $U(1)$-bundle associated with $\widetilde{L}$, we obtain a spin ${ }^{c}$ structure $\widetilde{t}$ on $c S$ that is an extension of $t$. We define $\delta(S, t)=w\left(S, c S, t_{c S}\right)$ by

$$
\delta(S, t)=-8 \delta^{\operatorname{Dirac}}(S, t)-\delta^{\operatorname{sign}}(S),
$$

where $\delta^{\operatorname{sign}}(S)$ (resp. $\delta^{\text {Dirac }}(S, t)$ ) is the contribution from the singularity of $c S$ to the index of the signature operator over $c S$ (resp. the $\operatorname{spin}^{c}$ Dirac operator over $c S$ associated with $\widetilde{t}$. Thus if a pair $(M, t)$ of a rational homology 3 -sphere and a $\operatorname{spin}^{c}$ structure on it bounds $\left(X, t_{X}\right)$, where $X$ is a compact 4-orbifold with $\operatorname{spin}^{c}$ structure $t_{X}$ and all the singular points of $X$ are isolated, we formally define the Fukumoto-Furuta invariant as

$$
w\left(M, X, t_{X}\right)=\sigma(X)+\sum_{\left(S,\left.t_{X}\right|_{S}\right)} \delta\left(S,\left.t_{X}\right|_{S}\right),
$$

where in the sum on the right hand side $S$ runs over all the links of the singularities of $X$ and $\left.t_{X}\right|_{S}$ is the $\operatorname{spin}^{c}$ structure of $S$ induced by $t_{X}$.

4.2. The equivariant eta invariants. The proof of the main theorem is deduced from the following proposition. 
Proposition 4.1. Let $S$ be a spherical 3-manifold with $\operatorname{spin}^{c}$ structure $t$, and $g$ be the metric of positive constant curvature on $S$. Then

$$
\eta^{\text {Dirac }}(S, t)=2 \delta^{\text {Dirac }}(S, t), \quad \eta^{\text {sign }}(S)=\delta^{\text {sign }}(S),
$$

where the eta invariants above are defined with respect to $g$.

The computation of the eta invariants goes in the same way as in [9]. So let us recall the theorem by Donnelly about the equivariant eta invariant. Let $\widetilde{Y}$ be a closed oriented smooth $n$-manifold ( $n$ odd) and $G$ be a finite group acting freely on $\tilde{Y}$. We assume that every $h \in G$ acts on $\tilde{Y}$ as an orientation-preserving diffeomorphism of $\widetilde{Y}$. Let $\widetilde{Y} \rightarrow Y=\widetilde{Y} / G$ be the associated unbranched $G$ covering, and $E_{\rho}=\tilde{Y} \times{ }_{\rho} \mathbf{C}^{k}$ be a flat $\mathbf{C}^{k}-$ bundle over $Y$ associated with some representation $\rho: G \rightarrow U(k)$. Now we consider the Dirac or the signature operator $D: \Gamma(W) \rightarrow \Gamma(W)$ over $Y$ (where $W$ is the corresponding spinor bundle), which is covered by a $G$-invariant operator $\widetilde{D}: \Gamma(\widetilde{W}) \rightarrow \Gamma(\widetilde{W})$ over $\widetilde{Y}$, where $\widetilde{W}$ is the spinor bundle covering $W$. We also consider the Dirac or the signature operator coupled with a flat connection $A_{\rho}$ on $E_{\rho}$,

$$
D_{\rho}: \Gamma\left(W \otimes E_{\rho}\right) \rightarrow \Gamma\left(W \otimes E_{\rho}\right) .
$$

Then we can define the usual eta function of $D_{\rho}$ of the form

$$
\eta_{\rho}(s, Y)=\sum_{\lambda \neq 0}(\operatorname{sgn} \lambda)|\lambda|^{-s},
$$

where the sum is taken over all the nonzero eigenvalues of $D_{\rho}$, and also the equivariant eta function with respect to $h \in G$ of the form

$$
\eta_{h}(s, \tilde{Y})=\sum_{\lambda \neq 0}(\operatorname{sgn} \lambda) \operatorname{Tr}\left(h_{\lambda}^{*}: E_{\lambda} \rightarrow E_{\lambda}\right)|\lambda|^{-s}
$$

where $E_{\lambda}$ is the $\lambda$-eigenspace of $\widetilde{D}$ and $h_{\lambda}^{*}$ is induced by $h$. Both of these functions can be extended analytically over 0 and let $\eta_{\rho}(Y)=\eta_{\rho}(0, Y)$ and $\eta_{h}(\widetilde{Y})=\eta_{h}(0, \widetilde{Y})$. Then we have

THEOREM $4.2([2])$.

$$
\eta_{\rho}(Y)=\frac{1}{|G|} \sum_{h \in G} \eta_{h}(\tilde{Y}) \chi_{\rho}(h),
$$

where $\chi_{\rho}$ is the character of $\rho$.

4.3. Proof of Proposition 4.1. We apply Theorem 4.2 to $\tilde{Y}=S^{3}$ and $Y=S=S^{3} / G$. Here for every $h \in G$, the fixed point set $\left(D^{4}\right)^{h}$ only consists of the origin 0 . If we denote by $p$ the order of $h$, then the quotient space $S^{3} /\langle h\rangle$ of $S^{3}$ by the cyclic group $\langle h\rangle$ generated by $h$ is a lens space of the form $L(p, q)$ for some $q$. It follows that for some appropriate choice of the coordinates $(z, w)$ of $D^{4} \subset \mathbf{C}^{2}$, we can assume that the action of $h$ on $D^{4}$ is given by

$$
h(z, w)=\left(\zeta^{k} z, \zeta^{k q} w\right) \quad(\zeta=\exp (2 \pi i / p))
$$

for some $k$. We extend the standard metric $g$ of $S^{3}$ to the metric $\widetilde{g}$ of $D^{4}$ of non-negative scalar curvature, and first we consider the case when $D$ is the Dirac operator. According to 4.1 , we fix one spin structure $c_{0}$ on $S$ and its unique extension $\widetilde{c}_{0}$ on $c S=D^{4} / G$, 
which is determined by the choice of the double covering $\sqrt{K}$ of the canonical bundle $K$ over $c S$. Here $K$ is the quotient space of some $G$-action on $\widetilde{K}=D^{4} \times \mathbf{C}$, which induces the covering $D^{4} \rightarrow c S=D^{4} / G$ of the base spaces. Hence $\sqrt{K}$ is determined by choosing a lift of the $G$-action on $\widetilde{K}$ to the double covering $\sqrt{\widetilde{K}}=D^{4} \times \mathbf{C} \rightarrow \widetilde{K}=D^{4} \times \mathbf{C}$ defined by $(z, w, \widetilde{w}) \rightarrow\left(z, w, \widetilde{w}^{2}\right)(\widetilde{w} \in \mathbf{C})$. For each $h \in G$, a lift of the action of $h$ on $\widetilde{K}$ to $\sqrt{\widetilde{K}}$, which we denote by $\widetilde{h}$, is represented as $\widetilde{h}(z, w, \widetilde{w})=\left(\zeta^{k} z, \zeta^{k q} w, \epsilon(h) \zeta^{k(-1-q) / 2} \widetilde{w}\right)$ for some choice of $\epsilon(h)= \pm 1$ (depending on $h$ ). Then $\widetilde{h}$ determines the lifts of the $h$ action to that on the \pm spinor bundles $\widetilde{W}^{ \pm}$over $D^{4}$ according to the representations $\widetilde{W}^{+}=\left(\wedge^{0,0} \oplus \wedge^{0,2}\right)\left(D^{4}\right) \otimes \sqrt{\widetilde{K}}$ and $\widetilde{W^{-}}=\wedge^{0,1}\left(D^{4}\right) \otimes \sqrt{\widetilde{K}}$. We also denote these actions by the same symbol $\widetilde{h}$. Then by the theorem of Donnelly [2], the $\widetilde{h}$-index $\operatorname{ind}(\widetilde{\mathcal{D}}, \widetilde{h})$ of the Dirac operator $\widetilde{\mathcal{D}}: \Gamma\left(\widetilde{W}^{+}\right) \rightarrow \Gamma\left(\widetilde{W}^{-}\right)$over $D^{4}$, which is given by

$$
\left.\operatorname{Tr} \widetilde{h}\right|_{\text {ker } \widetilde{\mathcal{D}}}-\left.\operatorname{Tr} \widetilde{h}\right|_{\text {ker } \widetilde{\mathcal{D}}}
$$

with the Atiyah-Patodi-Singer condition, is represented as follows.

$$
\operatorname{ind}(\widetilde{\mathcal{D}}, \widetilde{h})=\frac{\left.\operatorname{Tr} \widetilde{h}\right|_{(\widetilde{W}+)_{0}}-\left.\operatorname{Tr} \widetilde{h}\right|_{(\widetilde{W}-)_{0}}}{\operatorname{det}\left(1-(\widetilde{d})_{0}\right)\left(N_{0} \otimes \mathbf{C}\right)}-\frac{1}{2}\left(\eta_{\widetilde{h}}^{\operatorname{Dirac}}\left(S^{3}\right)+k_{\widetilde{h}}\right) .
$$

Here $N_{0}$ is the normal bundle of $\{0\} \subset D^{4}$ and the first term on the right hand side is the "Lefschetz number", i.e., the contribution from the unique fixed point $\{0\}$ to the $\widetilde{h}$-index, which we denote by $L(\widetilde{h}, \widetilde{\mathcal{D}})$, and $k_{\widetilde{h}}=\left.\operatorname{Tr} \widetilde{h}\right|_{\text {ker } \widetilde{D}}$ for $\widetilde{D}=\left.\widetilde{\mathcal{D}}\right|_{S^{3}}$. Since the metrics $g$ and $\widetilde{g}$ on $S^{3}$ and $D^{4}$ have non-negative (not identically zero) scalar curvature, we have

$$
\operatorname{ind}(\mathcal{D}, \widetilde{h})=0, \quad k_{\widetilde{h}}=0
$$

by the Weitzenböck formula, and hence

$$
\eta_{\widetilde{h}}^{\operatorname{Dirac}}\left(S^{3}\right)=2 L(\mathcal{D}, \widetilde{h}) .
$$

Next we consider a $\operatorname{spin}^{c}$ structure $t$ on $S$ such that the difference between $t$ and $c_{0}$ is given by a flat complex line bundle $L_{\rho}$ over $S$ with flat connection $A_{\rho}$ corresponding to some representation $\rho: G \rightarrow U(1)$, which extends to the flat line bundle $\widetilde{L}_{\rho}$ over $c S$. Then the $\operatorname{spin}^{c}$ Dirac operator corresponding to $t$ is equal to $D_{A_{\rho}}: \Gamma\left(W \otimes L_{\rho}\right) \rightarrow \Gamma\left(W \otimes L_{\rho}\right)$, covered by the $G$-invariant Dirac operator $\widetilde{D}: \Gamma(\widetilde{W}) \rightarrow \Gamma(\widetilde{W})$ over $S^{3}$, which also extends to the Dirac operator $\mathcal{D}: \Gamma\left(\widetilde{W}^{+}\right) \rightarrow \Gamma\left(\widetilde{W}^{-}\right)$over $D^{4}$. Here $W$ and $\widetilde{W}$ are the spinor bundles over $S$ and $S^{3}$ respectively, and $\widetilde{W}^{ \pm}$is the \pm spinor bundle over $D^{4}$ defined in the spin case, while for each $h \in G$ the lift of the $h$-action on $\widetilde{W}^{ \pm}$or $\widetilde{W}$, which we denote by $\widetilde{h}^{\prime}$, is defined as follows. If the order of $h$ is $p$ as before, $\rho(h)=\zeta^{a}$ for some $a \in \mathbf{Z}$. Hence $\widetilde{h}^{\prime}$ acts on $\sqrt{\widetilde{K}}$ by $\widetilde{h}^{\prime}(z, w, \widetilde{w})=\left(\zeta^{k} z, \zeta^{q k} w, \epsilon(h) \zeta^{k(-1-q) / 2+a} \widetilde{w}\right)$ so that the quotient of $\sqrt{\widetilde{K}}$ by $\widetilde{h}^{\prime}$ is $\sqrt{K} \otimes \widetilde{L}_{\rho}$. Thus the Lefschetz number $L\left(\widetilde{h}^{\prime}, \widetilde{\mathcal{D}}\right)$ is given by

$$
L\left(\widetilde{h}^{\prime}, \widetilde{\mathcal{D}}\right)=L(\widetilde{h}, \widetilde{\mathcal{D}}) \chi_{\rho}(h) .
$$

On the other hand by the theorem of Donnelly, the eta invariant $\eta^{\text {Dirac }}(S, t)$, which is equal to $\eta_{\rho}(S)$, satisfies

$$
\eta^{\text {Dirac }}(S, t)=\frac{1}{|G|} \sum_{h \in G} \eta_{\widetilde{h}}^{\text {Dirac }}\left(S^{3}\right) \chi_{\rho}(h)=\frac{1}{|G|} \sum_{h \in G, h \neq 1} \eta_{\widetilde{h}}^{\text {Dirac }}\left(S^{3}\right) \chi_{\rho}(h) .
$$


We note that $\eta^{\text {Dirac }}\left(S^{3}\right)$ (which is equal to $\eta_{\widetilde{h}}^{\text {Dirac }}\left(S^{3}\right)$ for $h=1$ ) is 0 since there is an orientation-reversing isometry of $S^{3}$. Here by the previous results we have

$$
\eta_{\widetilde{h}}^{\text {Dirac }}\left(S^{3}\right) \chi_{\rho}(h)=2 L(\widetilde{h}, \widetilde{\mathcal{D}}) \chi_{\rho}(h)=2 L\left(\widetilde{h}^{\prime}, \widetilde{\mathcal{D}}\right) .
$$

On the other hand the contribution $\delta^{\operatorname{Dirac}}(S, t)$ from $c S$ to the index of the Dirac operator over $c S$ with respect to $t_{c S}$ (which is an extension of $t$ as in 4.1) is given by

$$
\delta^{\text {Dirac }}(S, t)=\frac{1}{|G|} \sum_{h \in G, h \neq 1} L\left(\widetilde{h}^{\prime}, \widetilde{\mathcal{D}}\right)
$$

by the $\mathrm{V}$-index theorem. Thus we have the first claim of the proposition.

To prove the second claim of Proposition 4.1, we note that in [2] the $h$-signature $\operatorname{sign}\left(h, D^{4}\right)$ of $D^{4}$ for each $h \in G$ is represented as

$$
\operatorname{sign}\left(h, D^{4}\right)=\frac{\left.\operatorname{Tr} \widetilde{h}\right|_{\left(\wedge^{+}\right)_{0}}-\left.\operatorname{Tr} \widetilde{h}\right|_{\left(\wedge^{-}\right)_{0}}}{\operatorname{det}\left(1-(d \widetilde{h})_{0}\right)\left(N_{0} \otimes \mathbf{C}\right)}-\eta_{h}^{\operatorname{sign}}\left(S^{3}\right) .
$$

Here the actions of $h$ on $\wedge^{ \pm}$over $D^{4}$ and $\wedge^{e v}$ over $S^{3}$ induced by the original action on $D^{4}$ are also denoted by $h$. Since $\operatorname{sign}\left(h, D^{4}\right)=0$, the first term on the right hand side of the above equation, which comes from the $h$-index of the signature operator $\widetilde{\mathcal{D}}^{\text {sign }}$ over $D^{4}$ and we denote by $L\left(h, \widetilde{\mathcal{D}}^{\text {sign }}\right)$, is equal to $\eta_{h}^{\text {sign }}\left(S^{3}\right)$. For the above representation of the action of $h$, it is known that

$$
\eta_{h}^{\text {sign }}\left(S^{3}\right)=-\cot \frac{\pi k}{p} \cot \frac{\pi k q}{p} .
$$

Moreover we can see $([2])$ that

$$
\eta^{\text {sign }}(S)=\frac{1}{|G|} \sum_{h \in G} \eta_{h}^{\text {sign }}\left(S^{3}\right)=\frac{1}{|G|} \sum_{h \in G, h \neq 1} \eta_{h}^{\text {sign }}\left(S^{3}\right)=\frac{1}{|G|} \sum_{h \in G, h \neq 1} L\left(h, \widetilde{\mathcal{D}}^{\text {sign }}\right) .
$$

Here by the same reason as before we have $\eta^{\operatorname{sign}}\left(S^{3}\right)=0$, which yields the second equality above. Finally by the V-index theorem the last term of the above equation is also equal to the contribution $\delta^{\operatorname{sign}}(S)$ from $c S$ to the $V$-signature. This proves the second claim of the proposition.

4.4. Proof of Theorem 1.1. Let $(S, t)$ be a pair of a spherical 3 -manifold and a $\operatorname{spin}^{c}$ structure on it. We fix a metric $g$ of $S$ of positive constant curvature. Then by Rustamov's theorem we have

$$
\frac{1}{2} d(S, t)-\chi\left(H F_{\text {red }}(S, t)\right)=s w(S, t)+\frac{1}{8}\left(4 \eta^{\text {Dirac }}(S, t)+\eta^{\operatorname{sign}}(S)\right) .
$$

Here the 3-dimensional Seiberg-Witten invariant $s w(S, t)$ and the eta inavariants are defined with respect to $g$. Hence $s w(S, t)$ is zero since $g$ has positive scalar curvature, and by Proposition 4.1, we have

$$
\delta(S, t):=-\left(8 \delta^{\operatorname{Dirac}}(S, t)+\delta^{\operatorname{sign}}(S, t)\right)=-\left(4 \eta^{\operatorname{Dirac}}(S, t)+\eta^{\operatorname{sign}}(S, t)\right) .
$$

For the estimate of $\chi\left(H F_{\text {red }}(S, t)\right)$, we recall Némethi's result [12], where he observed a Heegaard Floer homology of a rational homology 3-sphere that bounds a negative definite plumbing $P(\Gamma)$ such that $\Gamma$ is an almost rational (AR) graph. Every link of a rational 
surface singularity and every Seifert rational homology 3-sphere (up to orientation) satisfy these conditions. In general we have

$$
\chi\left(H F_{\text {red }}(M, t)\right)=\operatorname{rank} H F_{\text {even }}(M, t)-\operatorname{rank} H F_{\text {odd }}(M, t)
$$

where $H F_{\text {odd }}$ and $H F_{\text {even }}$ denote the sets of elements of $H F_{\text {red }}$ with odd and even absolute $\mathbf{Z}_{2}$ grading respectively. We also note that $\chi(H F(-M, t))=-\chi(H F(M, t))$. If $M$ is a plumbed homology 3 -sphere satisfying the above conditions, then Theorem 8.3 in [12] shows that $H F_{\text {odd }}(-M, t)=0$ for any $\operatorname{spin}^{c}$ structure $t$ of $M$, and moreover we have

THEOREM 4.3 ([12]). Let $M$ be a link of a rational surface singularity. Then $H F_{\text {red }}(M, t)$ $=0$ for every $\operatorname{spin}^{c}$ structure of $M$.

It is well known that every spherical manifold $S$ is a link of a quotient singularity (up to orientation), and every such singularity is a rational surface singularity. Hence we have $H F_{\text {red }}(S, t)=0$ for every $\operatorname{spin}^{c}$ structure $t$ on $S$. It follows that

$$
4 d(S, t)=-\delta(S, t) .
$$

This proves Theorem 1.1.

Now we compare Theorem 2.9 with the Ozsváth-Szabó's theorem for a pair $(M, c)$ of a Seifert rational homology 3 -sphere and a spin structure on it. Suppose that $(M, c)$ bounds a negative definite spin 4 -manifold $\left(W, c_{W}\right)$. Then Theorem 2.9 shows that

$$
b_{2}(W) \equiv-\bar{\mu}(M, c) \quad(\bmod 16), \quad-\frac{\bar{\mu}(M, t)}{9} \leq b_{2}(W) \leq-\bar{\mu}(M, c),
$$

while Theorem 3.2 shows that

$$
b_{2}(W) \equiv 4 d(M, c) \quad(\bmod 8), \quad b_{2}(W) \leq 4 d(M, c) .
$$

If $M$ is non-spherical, the above two estimates are different in general, but Theorem 1.1 suggests that they derive similar results if $M$ is spherical. If a spherical 3-manifold $S$ is neither a lens space nor a Seifert fibration over $S^{2}(2,2, n)$ for some $n \geq 2$ (i.e., a prism manifold), we have $|\bar{\mu}(S, c)| \leq 8$ according to the list of $\bar{\mu}(S, c)=\delta(S, c)$ in [18]. It follows that the above two estimates lead to the same conclusion: $b_{2}(W)=-\bar{\mu}(S, c)=4 d(S, c)$. In fact we have the following result (which is the extension of Theorem 2 in [18]).

Corollary 4.4. Suppose that a pair $(M, c)$ of a Seifert rational homology 3-sphere and a spin structure on it bounds a negative definite spin 4-manifold $\left(W, c_{W}\right)$. If $|\bar{\mu}(M, c)| \leq 18$, then $b_{2}(W)=-\bar{\mu}(M, c)$.

REMARK 4.5. Strictly speaking, for the case when $S$ is a spherical manifold with $\bar{\mu}(S, c)=$ $-4 d(S, c)=-8$, Theorem 3.2 shows that either $b_{2}(W)=8$ or $b_{2}(W)=0$, while the estimate by Theorem 2.9 eliminates the second possibility. This case occurs if $S$ is a Seifert fibration over $S^{2}(2,3,5)$ with Seifert invariants $\{(2,1),(3,1),(5,10 k-4)\}$ for $k \geq 0$ ([18]), and $c$ is the unique spin structure. In such a case the Rokhlin invariant of $(S, c)$ is nonzero, and hence the case $b_{2}(W)=0$ is eliminated.

On the other hand, if $S$ is either a lens space or a prism manifold, the absolute value of $\bar{\mu}(S, c)=-4 d(S, c)$ may be arbitrary large. In fact in such a case, $\bar{\mu}(S, c)=\delta(S, c)$ is represented by a $\sigma$ function in $\S 2$. As in [5], if $p / q$ has a continued fraction expansion of 
the form $\left[a_{1}, \ldots, a_{s}\right]$ for $a_{i}$ even and $a_{i} \neq 0$, (in such a case $p$ and $q$ have opposite parity), then $\sigma(q, p,-1)=-\sum \operatorname{sgn}\left(a_{i}\right)$. Since $\bar{\mu}(L(p, q), c)=\sigma\left(q, p,(-1)^{c-1}\right)$, where $c=c(\mu)$ is chosen as in $\S 1$, there exist $p, q, c$ so that $-\bar{\mu}(L(p, q), c) \geq N$ for any given $N>0$ by some choice of $a_{i}$ 's above. The same statement is also true for the prism manifolds according to the list in [18]. In such cases the estimate given by Theorem 2.9 might be slightly stronger than that given by Theorem 3.2 .

4.5. Explicit computation for the lens spaces. In this subsection we focus on the lens space $L(p, q)$ with $\operatorname{gcd}(p, q)=1$ and $p>q>0$ and give more explicit relations between the two invariants in Theorem 1.1. First we compare the description of the $\operatorname{spin}^{c}$ structures $^{-}$ on the lens spaces given by [11] and the one described in $\S 2$. Let $(E, \alpha, \gamma)$ be the standard Heegaard diagram corresponding to the decomposition of $L(p, q)$ into two solid tori $U_{0}$ and $U_{1}$, where $\alpha$ (resp. $\gamma$ ) is the attaching circle of $U_{0}$ (resp. $U_{1}$ ). Here the Heegaard torus $E=\partial U_{0}=-\partial U_{1}$ is identified with $S^{1} \times S^{1}$ so that $\alpha$ corresponds to $S^{1} \times\{1$ point $\}$ and, if we put $\beta=\{1$ point $\} \times S^{1}$, the homology class of $\gamma$ in $E$ is represented by $p \beta-q \alpha$. Moreover if $L(p, q)$ is represented by the $-p / q$-surgery on the unknot of $S^{3}$, then $U_{0}$ is identified with the exterior of the unknot so that the meridian $\mu$ (resp. the preferred longitude $\lambda$ ) of the unknot corresponds to $\beta$ (resp. $\alpha$ ). Note that the orientation convention for the lens space in [11] is opposite to ours $(L(p, q)$ is described as $-L(p, q)$ in [11]). If we choose a base point $z$ on $E$, we have a map defined in [10]

$$
s_{z}: \alpha \cap \gamma \rightarrow \operatorname{spin}^{c}(L(p, q))
$$

from $\alpha \cap \gamma$ to the set of the $\operatorname{spin}^{c} \operatorname{structures} \operatorname{spin}^{c}(L(p, q))$ on $L(p, q)$, which is a bijection in the case of lens spaces. Furthermore in [11] Ozsváth-Szabó defined the circular ordering of $\operatorname{spin}^{c}(L(p, q)$, which is represented by a natural number in $\{0,1, \ldots, p-1\}$ as follows. First we consider the pointed Heegaard triple $(E, \alpha, \beta, \gamma, z)$, where $z$ is placed so that all the coefficients of the triply periodic domain connecting $\alpha, \beta$, and $\gamma$ are negative. Let $x_{0}, \ldots, x_{p-1}$ be the $p$ intersection points of $\alpha \cap \gamma$ which are placed in order along $\alpha$ so that $x_{p-1}$ is adjacent to $z$. Then the $\operatorname{spin}^{c}$ structure $s_{z}\left(x_{i}\right)$ is represented by $i(\bmod p)$. They also gave the following recursive formula.

Proposition 4.6 ([11], Proposition 4.8). For any integer $i$ with $0 \leq i<p+q$,

$$
d(L(p, q), i)=\left(\frac{p q-(2 i+1-p-q)^{2}}{4 p q}\right)-d(L(q, r), j) .
$$

Here $r$ and $j$ are the reductions modulo $q$ of $p$ and $i$ respectively.

In fact $(E, \alpha, \beta, \gamma)$ represents a compact 4 -manifold $X_{\alpha, \beta, \gamma}$ with

$$
\partial X_{\alpha, \beta, \gamma}=S^{3}-L(q, r)+L(p, q)
$$

where $S^{3}$ and $L(q, r)$ are represented by the Heegaard diagrams $(E, \alpha, \beta)$ and $(E, \beta, \gamma)$ respectively, and the intersection points $\beta \cap \gamma$ are placed in order along $\beta$ so that the corresponding $\operatorname{spin}^{c}$ structures (via $s_{z}$ as before) have the circular ordering as in the case of $L(p, q)$. Moreover it is shown in [11] that there are $p+q$ triangles $\phi_{i}(0 \leq i \leq$ $p+q-1)$ connecting the intersection points in $\alpha \cap \gamma$ and $\beta \cap \gamma$ corresponding to the $\operatorname{spin}^{c}$ structures with the same order $i$ (which is considered modulo $p$ and $q$ respectively), and the unique intersection point $\alpha \cap \beta$ (which corresponds to the unique $\operatorname{spin}^{c}$ structure 
on $S^{3}$ ). Furthermore as in [11], there is a correspondence (which we denote also by $s_{z}$ ) between the triangles $\phi_{i}$ and the $\operatorname{spin}^{c}$ structures on $X_{\alpha, \beta, \gamma}$ so that $s_{z}\left(\phi_{i}\right)$ induces the $i$-th $\operatorname{spin}^{c}$ structures $s_{z}\left(x_{i}\right)$ on $L(p, q)$ and $L(q, r)$. It is also shown in [11] that

$$
\left\langle c_{1}\left(s_{z}\left(\phi_{i}\right)\right), H\right\rangle=2 i+1-p-q
$$

where $H$ is the generator of $H_{2}\left(X_{\alpha, \beta, \gamma}\right)$. Hence the map $q: i \rightarrow p+q-1-i$ leaves the above value invariant, and in fact the correspondence $i \rightarrow q(i)$ represents the conjugation $t \rightarrow \bar{t}$ of the $\operatorname{spin}^{c}$ structures on $L(p, q)$ and $L(q, r)$ if the numbers are counted modulo $p$ and $q$ respectively (see also [8]). It follows that the $\operatorname{spin}^{c}$ structure $t$ on $L(p, q)$ comes from a spin structure (in this case $c_{1}(\operatorname{det} t)=t-\bar{t}=0$ ) if and only if $t$ corresponds to one of the integers in $\{(q-1) / 2,(p+q-1) / 2\}$ (just one of them is an integer if $p$ is odd and both of them are integers if $p$ is even).

Now we compare the representation of $\operatorname{spin}^{c}(L(p, q))$ above with that given in $\S 4.1$, where we describe the $\operatorname{spin}^{c}$ structure on $L(p, q)$ as the restriction to $L(p, q)$ of some $\operatorname{spin}^{c}$ structure on $c L(p, q)$. Note that the associated + spinor bundle is of the form $\left(1 \oplus K^{-1}\right) \otimes L$, where $K$ is the canonical line bundle and $L$ is some line bundle over $c L(p, q)$. We denote the restriction of the line bundle over $c L(p, q)$ to $L(p, q)$ by the same symbol. Note that the covering projection $\pi: S^{3} \rightarrow L(p, q)$ maps the curve $\left(\xi^{t}, \xi^{q t}\right)$ $(0 \leq t<1)$ in $S^{3}$ to the curve in $L(p, q)$ (which is the $-p / q$ surgery on the unknot) corresponding to the meridian $\mu$ of the unknot, which generates $\pi_{1}(L(p, q))$. Let $L_{\rho}$ be the flat line bundle over $L(p, q)$ corresponding to the representation $\rho: \pi_{1}(L(p, q)) \rightarrow U(1)$ defined by $\rho(\mu)=\zeta:=\exp (2 \pi i / p)$, i.e.,

$$
L_{\rho}=S^{3} \times \mathbf{C} / \widetilde{\rho}
$$

where $\widetilde{\rho}\left(z_{1}, z_{2}, w\right)=\left(\zeta z_{1}, \zeta^{q} z_{2}, \zeta w\right)$. Then every line bundle over $L(p, q)$ is of the form $L_{\rho}^{\otimes k}$ for some $k$, and $K=L_{\rho}^{\otimes(-q-1)}$. Moreover the $\operatorname{spin}^{c}$ structure on $L(p, q)$ corresponding to $\left(1 \oplus K^{-1}\right) \otimes L_{\rho}^{\otimes k}$ comes from a spin structure if and only if $L_{\rho}^{k}$ is the double covering $\sqrt{K}$ of $K$, in which case $k$ is either $-(q+1) / 2$ if $q$ is odd, or $-(p+q+1) / 2$ if $p+q$ is odd. Once we fix a spin structure $s_{0}$ corresponding to $\sqrt{K} \oplus \sqrt{K^{-1}}$ and denote by $t$ the $\operatorname{spin}^{c}$ structure on $L(p, q)$ corresponding to $\left(\sqrt{K} \oplus \sqrt{K^{-1}}\right) \otimes L_{\rho}^{\otimes \ell}$, then the conjugation $\bar{t}$ of $t$ corresponds to $\left(\sqrt{K} \oplus \sqrt{K^{-1}}\right) \otimes L_{\rho}^{\otimes(-\ell)}$.

In the meantime we have

Proposition 4.7. Let $\left\{x_{0}, \ldots, x_{p-1}\right\}$ be the intersection points $\alpha \cap \gamma$ for the pointed Heegaard diagram $(E, \alpha, \gamma, z)$ of $L(p, q)$ described and ordered as above. Then the difference $s_{z}\left(x_{i+k}\right)-s_{z}\left(x_{i}\right)$ is represented by $c_{1}\left(L_{\rho}^{\otimes k}\right) \in H^{2}(L(p, q), \mathbf{Z})$, where the subscripts are counted modulo $p$.

Proof. It is sufficient to prove the case where $k=1$. As is shown in [10], $s_{z}\left(x_{i+1}\right)-s_{z}\left(x_{i}\right)$ is the Poincaré dual of the homology class in $L(p, q)$ of a curve $\epsilon\left(x_{i}, x_{i+1}\right)$, which is the union of the curve in $\alpha$ from $x_{i}$ to $x_{i+1}$ and the curve in $\gamma$ from $x_{i+1}$ to $x_{i}$. Since the oriented curve $\gamma$ is represented by $(\exp (-2 \pi i q t), \exp (2 \pi i p t))(0 \leq t \leq 1)$ in $E=S^{1} \times S^{1}$ (where $\alpha=S^{1} \times\{*\}$ and $\beta=\{*\} \times S^{1}$ ), we see that $\epsilon\left(x_{i}, x_{i+1}\right)$ is $q^{*} \mu=q^{*} \beta$, where $q^{*}$ is the integer with $q q^{*} \equiv 1(\bmod p)$. On the other hand by the Poincaré duality and the universal coefficient theorem, $q^{*} \mu \in H_{1}(L(p, q), \mathbf{Z})$ is identified via $\operatorname{Ext}\left(H_{1}(L(p, q)), \mathbf{Z}\right)$ 
with the element $\widetilde{\rho}$ of $\operatorname{Hom}\left(H_{1}(L(p, q), \mathbf{Z}), \mathbf{Q} / \mathbf{Z}\right)$ defined by

$$
\widetilde{\rho}(c)=\mathrm{lk}_{\mathbf{Q} / \mathbf{Z}}\left(q^{*} \mu, c\right)
$$

for $c \in H_{1}(L(p, q), \mathbf{Z})$, where $\mathrm{lk}_{\mathbf{Q} / \mathbf{Z}}$ denotes the linking form over $H_{1}(L(p, q), \mathbf{Z})$. Since

$$
\mathrm{lk}_{\mathbf{Q} / \mathbf{Z}}(\mu, \mu)=q / p \quad(\bmod 1)
$$

for the meridian $\mu$, we see that $c_{1}\left(L_{\rho}\right)$ is the Poincaré dual of $q^{*} \mu$, which gives the desired result.

Thus if the spin structure on $L(p, q)$ given by some choice of $\sqrt{K}$ is labelled by $s$, then the $\operatorname{spin}^{c}$ structure corresponding to $\left(\sqrt{K} \oplus \sqrt{K^{-1}}\right) \otimes L_{\rho}^{k}$ is labelled by $s+k$, and its conjugate is labelled by $s-k$ (modulo $p$ ). The value of the correction term for $L(p, q)$ with spin structure is related to the $\sigma$-function as follows.

Proposition 4.8. Let $p, q$ be the integers with $\operatorname{gcd}(p, q)=1$ and $p>q>0$. If $q$ is odd, then

$$
4 d\left(L(p, q), \frac{q-1}{2}\right)=-\sigma(q, p, 1),
$$

while if $p$ and $q$ have opposite parity, then

$$
4 d\left(L(p, q), \frac{p+q-1}{2}\right)=-\sigma(q, p,-1) .
$$

Proof. The case where $p$ is odd comes from Theorem 1.1 since the spin structure on $L(p, q)$ is unique. Note that in the description of $\bar{\mu}(L(p, q), c)$ in terms of $\sigma(q, p, \epsilon)$, the last term $\epsilon$ is determined so that $\epsilon=1$ if $\sqrt{K}$ is $L_{\rho}^{\otimes(-(1+q) / 2)}$ and $\epsilon=-1$ if $\sqrt{K}$ is $L_{\rho}^{\otimes(-(p+q+1) / 2)}$. Suppose that $p$ is even. Then we already have

$$
4 d\left(L(q, p), \frac{p+q-1}{2}\right)=-\sigma(p, q,-1)
$$

since $q$ is odd and hence $(p+q-1) / 2(\bmod q)$ and -1 are the unique choices for the spin structure on $L(q, p)$ on the both sides. Strictly speaking, the number $p$ in the above equation should be replaced by its residue $r$ modulo $q$. Suppose that $p=n q+r$ for some $n>0$. If $n$ is even, then the above equation is the same as that obtained by replacing $p$ by $r$ on both sides. If $n$ is odd, then the above equation is equivalent to

$$
4 d\left(L(q, r), \frac{r-1}{2}\right)=-\sigma(r, q, 1),
$$

which appeared in the first claim since $\sigma(n q+r, q, \epsilon)=\sigma\left(r, q,(-1)^{n} \epsilon\right)$. Then the claim for $p$ follows by comparing the formula in Proposition 4.6 with that given in $\S 2$, i.e.,

$$
\sigma(p, q,-1)+\sigma(q, p,-1)=-\operatorname{sgn} p q=-1 .
$$

In fact, this reciprocity is the same as the Ozsváth-Szabó's recursive formula for this particular case.

The explicit correspondence between the correction term $d$ and $\bar{\mu}$ for lens spaces with general $\operatorname{spin}^{c}$ structure is given as follows.

Remark 4.9. Consider a spin structure on $L(p, q)(p>q>0)$ such that its unique extension on $c L(p, q)$ has the + spinor bundle of the form $\sqrt{K} \oplus \sqrt{K^{-1}}$. Here we put $\epsilon=1$ 
if $\sqrt{K}=L_{\rho}^{\otimes(-(q+1) / 2)}$ and $\epsilon=-1$ if $\sqrt{K}=L_{\rho}^{\otimes(-(p+q+1) / 2)}$. We note that in Proposition 4.8 the first and the second cases correspond to $(q-1) / 2$ and $(p+q-1) / 2(\bmod p)$ respectively in Ozsváth-Szabó's notation. Once we choose such a spin structure (and $\sqrt{K})$, any $\operatorname{spin}^{c}$ structure $t$ on $L(p, q)$ is a restriction of that on $c L(p, q)$ whose + spinor bundle is given by $\left(\sqrt{K} \oplus \sqrt{K^{-1}}\right) \otimes L_{\rho}^{\otimes a}$ for some $a$. Then we can compute $4 d(L(p, q), t)=$ $-\bar{\mu}(L(p, q), t)$ explicitly by using the formula in $\S 4.3$ (see also the computation in [5]) as follows. First let us recall that

$$
4 d(L(p, q), t)=\delta^{\operatorname{sign}}(L(p, q))+8 \delta^{\text {Dirac }}(S, t) .
$$

Here the first term is equal to

$$
\frac{1}{p} \sum_{k=1}^{p-1}\left(-\cot \frac{\pi k}{p} \cot \frac{\pi k q}{p}\right)
$$

(which is the Dedekind sum up to multiplicative constant), while the second term is equal to

$$
\frac{1}{p} \sum_{k=1}^{p-1}\left(-2 \epsilon^{k} \csc \frac{\pi k}{p} \csc \frac{\pi k q}{p} \exp \left(\frac{2 \pi i k a}{p}\right)\right)=\frac{1}{p} \sum_{k=1}^{p-1}\left(-2 \epsilon^{k} \csc \frac{\pi k}{p} \csc \frac{\pi k q}{p} \cos \frac{2 \pi k a}{p}\right) .
$$

By Proposition 4.7 we see that $d(L(p, q), t)=d\left(L(p, q), a+\frac{q-1}{2}\right)$ if $\sqrt{K}=L_{\rho}^{\otimes(-(q+1) / 2)}$ $(\epsilon=1)$, and $d(L(p, q), t)=d\left(L(p, q), a+\frac{p+q-1}{2}\right)$ if $\sqrt{K}=L_{\rho}^{\otimes(-(p+q+1) / 2)}(\epsilon=-1)$. Since $\cos \left(2 \pi\left(a+\frac{p}{2}\right) / p\right)=-\cos (2 \pi a / p)$, in either case we have

$$
4 d(L(p, q), i)=-\frac{1}{p}\left(\sum_{k=1}^{p-1} \cot \frac{\pi k}{p} \cot \frac{\pi k q}{p}+2 \csc \frac{\pi k}{p} \csc \frac{\pi k q}{p} \cos \frac{2 \pi k\left(i-\frac{q-1}{2}\right)}{p}\right) .
$$

The above explicit formula is also obtained by Tange [17] by using the formulae for the Dedekind sums and for the correction term deduced from Proposition 4.6 (without using the index theorem).

5. Concluding remarks. A Seifert rational homology 3-sphere is a boundary of a plumbing $P(\Gamma)$ for some weighted tree $\Gamma$. In particular $L(p, q)$ is a boundary of a negative definite plumbing $P(\Gamma)$, where $\Gamma$ is of the form

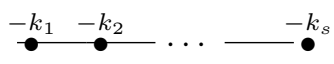

and

$$
\frac{p}{q}=\left[k_{1}, \ldots, k_{s}\right]:=k_{1}-\frac{1}{\cdots-\frac{1}{k_{s}}}
$$

with $k_{i} \geq 2$ for all $i$. Let $b_{j}$ be the generator of $L:=H_{2}(P(\Gamma), \mathbf{Z})$ corresponding to the $j$ th vertex of $\Gamma, g_{j}$ be the dual generator of $L^{\prime}:=H_{2}(P(\Gamma) . \partial P(\Gamma), \mathbf{Z})$, and $\left[g_{j}\right]=\partial g_{j} \in$ $H_{1}(L(p, q), \mathbf{Z})$. Then if we write

$$
\frac{n_{i, j}}{n_{i+1, j}}=\left[k_{i}, \ldots, k_{j}\right]
$$


(we have $p=n_{1, s}$ and $q=n_{2, s}$ ), as in [12] we have

$$
\left[g_{j}\right]=n_{j+1, s}\left[g_{s}\right] .
$$

(We put $n_{s+1, s}=1$ and $n_{s+2, s}=0$.) As is shown in [12], for any $\operatorname{spin}^{c}$ structure $s$ on $L(p, q)$ there exists a unique distinguished representative in $L^{\prime}$ for $s$, which is defined as follows. First note that $s$ is a restriction of a $\operatorname{spin}^{c}$ structure $\widetilde{s}$ on $P(\Gamma)$, which is uniquely determined by $c_{1}(\operatorname{det} \widetilde{s})$ since $P(\Gamma)$ is 1 -connected. Here $c_{1}(\operatorname{det} \widetilde{s})$ is represented as $K_{\Gamma}+2 \ell_{r} \in L^{\prime}$ via Poincaré duality, where $K_{\Gamma}$ is the canonical class of $P(\Gamma)$ defined by $K_{\Gamma}=\sum_{j}\left(k_{j}-2\right) g_{j}$ and $\ell_{r}=-\sum_{j} a_{j} g_{j}$ for some $a_{j}$. Then the images of them in $H_{1}(L(p, q), \mathbf{Z})$ are given by

$$
\left[K_{\Gamma}\right]=\sum_{j}\left(k_{j}-2\right) n_{j+1, s}\left[g_{s}\right]=\sum_{j=1}^{s}\left(n_{j, s}-2 n_{j+1, s}+n_{j+2, s}\right)\left[g_{s}\right]=(p-q-1)\left[g_{s}\right]
$$

and

$$
\left[\ell_{r}\right]=-\sum_{j=1}^{s} a_{j} n_{j+1, s}\left[g_{s}\right]=:-a\left[g_{s}\right] .
$$

Then according to [12] $K_{\Gamma}+2 \ell_{r}$ is distinguished if and only if the coefficients of $\ell_{r}$ satisfy

$$
\begin{aligned}
(S I) \quad a_{j} \geq 0, \quad j=1, \ldots, s \\
n_{j s}>\sum_{i=j}^{s} n_{i+1, s} a_{i} \quad j=1, \ldots, s .
\end{aligned}
$$

We note that if $L(p, q)$ is represented as the $-p / q$ surgery on the unknot, $\left[g_{1}\right]$ corresponds to the generator of $H_{1}(L(p, q), \mathbf{Z}) \cong \mathbf{Z}_{p}$ represented by the meridian of the unknot. Furthermore since $\left[g_{1}\right]=n_{2, s}\left[g_{s}\right]=q\left[g_{s}\right]$ we have $\left[g_{s}\right]=q^{\prime}\left[g_{1}\right]$, where $q^{\prime}:=n_{1, s-1}$ satisfies $q q^{\prime} \equiv 1(\bmod p)$.

Now we compare the distinguished representative for $s$ with the representation of $s$ given in $\S 4$. We denote by $L_{\rho}$ the line bundle over $L(p, q)$ defined by $L_{\rho}=S^{3} \times \mathbf{C} / \mathbf{Z}_{p}$ where the generator $g$ of $\mathbf{Z}_{p}$ acts on $D^{4} \times \mathbf{C}$ by

$$
g\left(z_{1}, z_{2}, w\right)=\left(\eta z_{1}, \eta^{q} z_{2}, \eta w\right) \quad(\eta=\exp (2 \pi i / p)) .
$$

Then if we idetify $H_{1}(L(p, q), \mathbf{Z})$ with $H^{2}(L(p, q), \mathbf{Z})$ via the Poincaré duality we see that

$$
c_{1}\left(L_{\rho}\right)=q^{\prime}\left[g_{1}\right]=\left[g_{s}\right] .
$$

Hence if we denote by $\widetilde{s}_{\text {can }}$ the $\operatorname{spin}^{c}$ structure on $c L(p, q)$ whose determinant line bundle is the canonical line bundle $K$ over $c L(p, q)$, we see that

$$
\left.c_{1}\left(\operatorname{det} \widetilde{s}_{\mathrm{can}}\right)\right|_{L(p, q)}=c_{1}\left(L_{\rho}^{\otimes(-q-1)}\right)=-(q+1)\left[g_{s}\right]
$$

It follows from the above formula that the $\operatorname{spin}^{c}$ structure $s_{\text {can }}$ on $L(p, q)$ induced by $\widetilde{s}_{\text {can }}$ coincides with that corresponding to $K_{\Gamma}$. Moreover using Proposition 4.8 we can see the following proposition.

Proposition 5.1. For $s_{\text {can }}$ on $L(p, q), d\left(L(p, q), s_{\text {can }}\right)=d(L(p, q),-1)$ in Oszváth-Szabó's notation. For the $\operatorname{spin}^{c}$ structure $s$ on $L(p, q)$ represented by $K+2 \ell_{r}$ defined above, which coincides with $s_{\text {can }} \otimes L_{\rho}^{\otimes(-a)}$, we have $d(L(p, q), s)=d(L(p, q),-1-a)$. 
Finally we point out that the claim (for spin structures) in Theorem 1.1 also holds for certain plumbed 3-manifolds as follows. The proof will be discussed elsewhere.

TheOREM 5.2 ([20]). Let $M$ be a boundary of a negative definite plumbing $P(\Gamma)$. Suppose that $m_{v} \leq-2$ for any vertex $v$ of $\Gamma$ and $m_{v} \leq-\nu_{v}$ for any $v$ with $\nu_{v} \geq 3$. Here $m_{v}$ is the weight of $v$ and $\nu_{v}$ is the number of edges in $\Gamma$ connected with $v$. Then for any spin structure $c$ on $M,-4 d(M, c)$ coincides with the Neumann-Siebenmann invariant of $(M, c)$.

We note that any plumbed 3-manifold satisfying the conditions in Theorem 5.2 is a link of a rational surface singularity $([12])$ as in the cases of spherical 3-manifolds. But not all spherical 3-manifolds satisfy the above condition.

Acknowledgments. The author thanks the referee for careful reading of the first draft of this paper. After having submitted this paper, the author noticed the paper by Stipsicz [16], which claims that the correction term coincides with the $\bar{\mu}$-invariant (up to constant) for links of rational surface singularities. All the 3-manifolds treated in this paper and [20] are contained in the class of the above types. But our method is completely different from [16], and the results of this paper are still of some independent interest.

\section{References}

[1] S. K. Donaldson, An application of gauge theory to four-dimensional topology, J. Diff. Geom. 18 (1983), 279-315.

[2] H. Donnelly, Eta invariants for G-spaces, Indiana Univ. Math. J. 27 (1978), 889-918.

[3] M. Furuta, Monopole equations and the 11/8-conjecture, Math. Res. Lett. 8 (2001), 289291.

[4] Y. Fukumoto and M. Furuta, Homology 3-spheres bounding acyclic 4-manifolds, Math. Res. Lett. 7 (2000), 757-766.

[5] Y. Fukumoto, M. Furuta, and M. Ue, $W$ invariants and the Neumann-Siebenmann invariants for Seifert homology 3-spheres, Topol. Appl. 116 (2001), 333-369.

[6] M. Furuta and Y. Kametani, Equivariant maps between sphere bundles over tori and $K O^{*}$-degree, math.GT/0502511 (2005).

[7] T. Kawasaki, The index of elliptic operators over $V$-manifolds, Nagoya Math. J. 84 (1981), 135-157.

[8] B. Owens and S. Strle, Rational homology spheres and the four-ball genus of knots, Adv. in Math. 200 (2006), 196-216.

[9] P. Ozsváth and Z. Szabó, The theta divisor and the Casson-Walker invariant, math.GT/0006194 (2000).

[10] P. Ozsváth and Z. Szabó, Holomorphic disks and topological invariants for closed threemanifolds, Ann. of Math. 159 (2004), 1027-1158.

[11] P. Ozsváth and Z. Szabó, Absolutely graded Floer homologies and intersection forms for four manifolds, Adv. in Math. 173 (2003), 179-261.

[12] A. Némethi, On the Ozsváth-Szabó invariant of negative definite plumbed 3-manifolds, Geo. Topol. 9 (2005), 991-1042.

[13] L. I. Nicolaescu, Seiberg-Witten invariants of rational homology 3-spheres, Commun. Contemp. Math. 6 (2004), 833-806. 
[14] R. Rustamov, A surgery formula for renormalized Euler characteristic of Heegaard Floer homology, math.GT/0409294 (2004).

[15] N. Saveliev, Fukumoto-Furuta invariants of plumbed homology 3-spheres, Pacific J. Math. 205 (2002), 465-490.

[16] A. I. Stipsicz, On the $\bar{\mu}$-invariant of rational surface singularities, Proc. Amer. Math. Soc. 136 (2008), 3815-3823.

[17] M. Tange, On applications of correction term formula of lens spaces, preprint, 2007.

[18] M. Ue, On the intersection forms of spin 4-manifolds bounded by spherical 3-manifolds, Alg. Geom. Topol. 1 (2001), 549-578.

[19] M. Ue, The Neumann-Siebenmann invariant and Seifert surgery, Math. Z. 250 (2005), 475-493.

[20] M. Ue, The Ozsváth-Szabó and the Neumann-Siebenmann invariants for certain plumbed 3-manifolds, preprint, Kyoto Univ., 2007. 
